\title{
A SPACE-TIME FINITE ELEMENT METHOD FOR NEURAL FIELD EQUATIONS WITH TRANSMISSION DELAYS*
}

\author{
MÓNIKA POLNER ${ }^{\dagger}$, J. J. W. VAN DER VEGT ${ }^{\ddagger}$, AND S. A. VAN GILS ${ }^{\S}$
}

\begin{abstract}
We present and analyze a new space-time finite element method for the solution of neural field equations with transmission delays. The numerical treatment of these systems is rare in the literature and currently has several restrictions on the spatial domain and the functions involved, such as connectivity and delay functions. The use of a space-time discretization, with basis functions that are discontinuous in time and continuous in space (dGcG-FEM), is a natural way to deal with space-dependent delays, which is important for many neural field applications. In this paper we provide a detailed description of a space-time dGcG-FEM algorithm for neural delay equations, including an a priori error analysis. We demonstrate the application of the dGcG-FEM algorithm on several neural field models, including problems with an inhomogeneous kernel.
\end{abstract}

Key words. neural fields, transmission delays, discontinuous Galerkin, finite element methods, space-time methods

AMS subject classifications. 65M60, 65M15, 65R20, 37M05, 92C20

DOI. $10.1137 / 16 \mathrm{M} 1085024$

1. Introduction. The motivation of this work is the need for numerical methods that can accurately and efficiently discretize delayed integro-differential equations originating from neural field models, in particular when the delay in the system is space dependent. Only a few studies so far have considered the numerical treatment of neural field systems; see [8], [10], [11], and references therein. In [8], the authors used special types of delay and connectivity functions in order to reduce the spatial discretization to a large system of delay differential equations with constant time delays. This system was then solved with the MATLAB solver dde23. In [10] a new numerical scheme was introduced that includes a convolution structure and hence allows the implementation of fast numerical algorithms. In both studies the connectivity kernel depends on the distance between two spatial locations. While this choice has been shown to successfully model neural activity known from experiments, it introduces, however, a limitation to the applicability of the presented techniques.

Here we propose the use of space-time finite element methods (FEMs) using discontinuous basis functions in time and continuous basis functions in space (dGcGFEM), which are well established to solve ordinary and partial differential equations; see, e.g., [5], [6], [7], [9], [12], [13]. The novelty of this work is the successful application of the space-time dGcG method to the neural field equations. The motivation of our choice is that the time-discontinuous Galerkin method has good long-time accuracy

*Submitted to the journal's Computational Methods in Science and Engineering section July 15, 2016; accepted for publication (in revised form) July 18, 2017; published electronically September $14,2017$.

http://www.siam.org/journals/sisc/39-5/M108502.html

Funding: The first author's work was supported by the Hungarian Scientific Research Fund, grant K109782. The ELI-ALPS project (GINOP-2.3.6-15-2015-00001) is supported by the European Union and co-financed by the European Regional Development Fund.

${ }^{\dagger}$ Bolyai Institute, University of Szeged, H-6720 Szeged, Aradi vértanúk tere 1, Hungary, and ELIALPS, ELI-HU Non-Profit Ltd, Dugonics tér 13, Szeged 6720, Hungary (polner@math.u-szeged.hu).

${ }^{\ddagger}$ Mathematics of Computational Science Group, Department of Applied Mathematics, University of Twente, P.O. Box 217, 7500 AE, Enschede, The Netherlands (j.j.w.vandervegt@utwente.nl).

$\S$ Applied Analysis, Department of Applied Mathematics, University of Twente, P.O. Box 217, 7500 AE, Enschede, The Netherlands (s.a.vangils@utwente.nl).

B797 
[6], [12]. Moreover, the use of a space-time discretization is a natural way to deal with the space-dependent delays. As will be discussed later, there is no need in a space-time method to interpolate the solution from previous time levels. The spacetime dGcG method was successfully used for stiff systems and is well suited for mesh adaptation, which is of great importance when local changes in the solution are of interest. Further benefits are that we do not need to make restrictions, neither to the functions involved in the system, such as the connectivity kernel or the delay function, nor to the dimension or shape of the spatial domain.

In this paper we present a novel space-time dGcG method for delay differential equations. We provide a theoretical analysis of the stability and order of accuracy of the numerical discretization and demonstrate its application on a number of neural field problems. We focus on the design and an a priori error analysis of the space-time dGcG-FEM for nonlinear neural field equations with space-dependent delay.

The outline of this paper is as follows. In the introductory section 2 we recall a mathematical model for neural fields. In section 3 we introduce the space-time dGcGFEM method. The main difficulty is the treatment of the delay term in the neural field equations, which is investigated in detail in section 3.2. An a priori error analysis of the space-time discretization is given in section 4 . Next, we show in section 5 some numerical simulations for the neural field equations in one and two space dimensions with one population. Some of the examples are taken from literature [8], [14], where both analytical and numerical results are known for comparison. We demonstrate some further computational benefits of the space-time dGcG-FEM by introducing an inhomogeneous kernel in the delay term in section 5.2.1. The numerical algorithms presented in [8] and [10] are not suitable for the treatment of local inhomogeneities.

In consecutive papers we will show computations on more complicated spatial domains and extend the model to more populations in the neural field system.

2. Neural fields with space-dependent delays. The mathematical model for neural fields with space-dependent delays is as follows. Consider $p$ populations consisting of neurons distributed over a bounded, connected, and open domain $\Omega \subset \mathbb{R}^{d}$, $d=1,2,3$. For each $i$, the variable $V_{i}(t, r)$ is the membrane potential at time $t$, averaged over those neurons in the $i$ th population positioned at $r \in \Omega$. These potentials are assumed to evolve according to the following system of integro-differential equations:

$$
\frac{\partial V_{i}}{\partial t}(t, r)=-\alpha_{i} V_{i}(t, r)+\sum_{j=1}^{p} \int_{\Omega} J_{i j}\left(r, r^{\prime}, t\right) S_{j}\left(V_{j}\left(t-\tau_{i j}\left(r, r^{\prime}\right), r^{\prime}\right)\right) d r^{\prime}
$$

for $i=1, \ldots, p$. The intrinsic dynamics exhibits exponential decay to the baseline level 0 , as $\alpha_{i}>0$. The propagation delays $\tau_{i j}\left(r, r^{\prime}\right)$ measure the time it takes for a signal sent by a type- $j$ neuron located at position $r^{\prime}$ to reach a type- $i$ neuron located at position $r$. The function $J_{i j}\left(r, r^{\prime}, t\right)$ represents the connection strength between population $j$ at location $r^{\prime}$ and population $i$ at location $r$ at time $t$. The firing rate functions are $S_{j}$. For the definition and interpretation of these functions we refer to [15]. Some examples will be given in later sections.

Throughout this paper we consider a single population, $p=1$, in a bounded domain $\Omega \subset \mathbb{R}^{d}$, on a time interval $\left[t_{0}, T\right)$, with $T>t_{0}$ the final time,

$$
\frac{\partial u}{\partial t}(t, x)=-\alpha u(t, x)+\int_{\Omega} J(x, r) S(u(t-\tau(x, r), r)) d r, \quad \alpha>0 .
$$

Note that we will only deal with autonomous systems. Therefore, we assume from here on that the connectivity does not depend on time. We assume that the following 
hypotheses are satisfied for the functions involved in the system (as in [14]): the connectivity kernel $J \in C(\bar{\Omega} \times \bar{\Omega})$, the firing rate function $S \in C^{\infty}(\mathbb{R})$ and its $k$ th derivative is bounded for every $k \in \mathbb{N}_{0}$, and the delay function $\tau \in C(\bar{\Omega} \times \bar{\Omega})$ is nonnegative.

Without loss of generality, we take $t_{0}=0$. From the assumption on the delay function $\tau$, we may set

$$
0<\tau_{\max }=\sup _{(x, r) \in \bar{\Omega} \times \bar{\Omega}} \tau(x, r)<\infty .
$$

Note that when $\tau_{\max }=0$, the delay function $\tau(x, r)=0$ for all $(x, r) \in \bar{\Omega} \times \bar{\Omega}$, and in this case (2) reduces to an integro-differential equation without delay. As we will see later, our numerical method can handle this case as well.

Let $Y=C(\bar{\Omega})$ and set $X=C\left(\left[-\tau_{\max }, 0\right] ; Y\right)$. For $\varphi \in X, s \in\left[-\tau_{\max }, 0\right]$ and for $x \in \Omega$ we write $\varphi(s)(x)=\varphi(s, x)$, and its norm is given by

$$
\|\varphi\|_{X}=\sup _{s \in\left[-\tau_{\max }, 0\right]}\|\varphi(s, \cdot)\|_{Y}
$$

where $\|\varphi(s, \cdot)\|_{Y}=\sup _{x \in \Omega}|\varphi(s, x)|$. From the assumption on the connectivity kernel, it follows that it is bounded in the following norm:

$$
\|J\|_{C}=\sup _{(x, r) \in \bar{\Omega} \times \bar{\Omega}}|J(x, r)| .
$$

We use the traditional notation for the state of the system at time $t$ :

$$
u_{t}(s)=u(t+s) \in C(\bar{\Omega}), \quad s \in\left[-\tau_{\max }, 0\right], t \geq 0 .
$$

Define the nonlinear operator $G: X \rightarrow Y$ by

$$
G(\varphi)(x)=\int_{\Omega} J(x, r) S(\varphi(-\tau(x, r), r)) d r .
$$

Then the neural field equation (2) can be written as a delay differential equation (DDE) as

$$
\frac{\partial u}{\partial t}(t)=-\alpha u(t)+G\left(u_{t}\right)
$$

where the solution is an element of $C\left(\left[-\tau_{\max }, \infty\right) ; Y\right) \cap C^{1}([0, \infty) ; Y)$. Similarly, we have the state of the solution at time $t$ defined as $u_{t}(s)(x)=u(t+s, x), s \in$ $\left[-\tau_{\max }, 0\right], t \geq 0, x \in \Omega$. It was shown in [14] that under the above assumptions on the connectivity, the firing rate function and delay, the operator $G$ is well defined and satisfies a global Lipschitz condition.

Note that the assumptions on the firing rate function $S$ imposed in [14] were needed for further analysis of the neural field equations. For the numerical analysis presented in this paper it is sufficient to assume that $S$ is Lipschitz continuous.

3. The discontinuous Galerkin finite element method. The starting point of our numerical discretization is the weak formulation. The numerical method is investigated for the nonlinear equation (4), which may be written in variational form as follows: Find $u \in C^{1}([0, T), Y) \cap C\left(\left[-\tau_{\max }, T\right), Y\right)$ such that

$$
\begin{array}{lr}
\left(\frac{\partial u}{\partial t}(t)+\alpha u(t), v\right)-\left(G\left(u_{t}\right), v\right)=0 & \forall v \in Y, \forall t \in(0, T), \\
u(s)=u_{0}(s), & s \in\left[-\tau_{\max }, 0\right],
\end{array}
$$




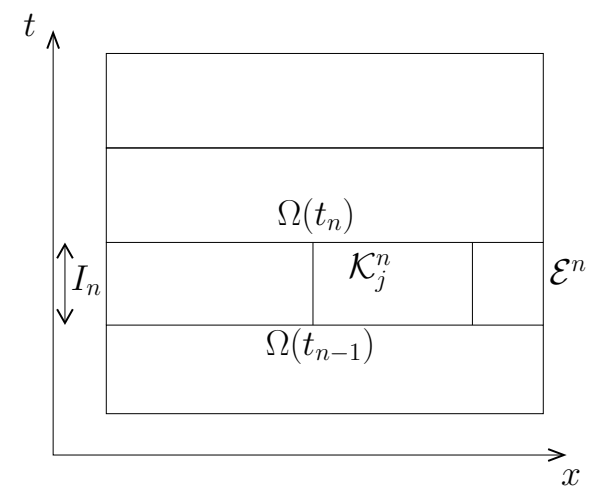

FIG. 1. Two-dimensional space-time elements in physical space.

where $(\cdot, \cdot)$ is the usual $L^{2}(\Omega)$ inner product. Here the delay contribution is expressed as

$$
\left(G\left(u_{t}\right), v\right)=\int_{\Omega} G\left(u_{t}\right)(x) v(x) d x=\int_{\Omega} \int_{\Omega} J(x, r) S\left(u_{t}(-\tau(x, r), r)\right) d r v(x) d x .
$$

Note that for any $t>0$, all functions in the inner product are elements of $Y=C(\bar{\Omega})$, which is a dense subset of $L^{2}(\Omega)$, and hence the inner product is well defined.

3.1. The space-time dGcG-FEM discretization. Consider the neural field equations in the domain $\Omega$. We will not distinguish between space and time variables and consider directly the space $\mathbb{R}^{d+1}$, where $d$ is the number of space dimensions.

Let $\mathcal{E} \subset \mathbb{R}^{d+1}$ be an open, bounded, space-time domain in which a point has coordinates $(t, x) \in \mathbb{R}^{d+1}$, with $x \in \mathbb{R}^{d}$ the position vector and time $t$. First, partition the time interval $\bar{I}=[0, T]$ using the time levels $0=t_{0}<t_{1}<\cdots<t_{N}=T$ and denote by $I_{n}=\left(t_{n-1}, t_{n}\right]$ the $n$th time interval of length $k_{n}=t_{n}-t_{n-1}$. A space-time slab is defined as $\mathcal{E}^{n}=I_{n} \times \Omega$. Second, we approximate the spatial domain $\Omega$ with $\Omega_{h}$ using a tessellation of nonoverlapping hexahedral elements (line elements in 1D, quadrilaterals in $2 \mathrm{D}$, etc.)

$$
\overline{\mathcal{T}}_{h}=\left\{K_{j}: \bigcup_{j=1}^{M} \bar{K}_{j}=\bar{\Omega}_{h}, K_{j} \cap K_{i}=\emptyset \text { if } i \neq j\right\} .
$$

The domain approximation is such that $\Omega_{h} \rightarrow \Omega$ as $h \rightarrow 0$, where $h$ is the radius of the smallest sphere containing each element $K_{j} \in \overline{\mathcal{T}}_{h}$. The space-time elements $\mathcal{K}_{j}^{n}$ are now obtained as $\mathcal{K}_{j}^{n}=\left(t_{n-1}, t_{n}\right) \times K_{j}$. The space-time tessellation is defined as

$$
\mathcal{T}_{h}^{n}=\left\{\mathcal{K}=G_{\mathcal{K}}^{n}(\hat{\mathcal{K}}): K \in \overline{\mathcal{T}}_{h}\right\}
$$

where $G_{\mathcal{K}}^{n}$ denotes the mapping from the space-time reference element $\hat{\mathcal{K}}=(-1,1)^{d+1}$ to the space-time element in physical space $\mathcal{K}$; see Figure 1 . The tessellation $\mathcal{T}_{h}$ of the whole discrete space-time domain is $\mathcal{T}_{h}=\cup_{n=1}^{N} \mathcal{T}_{h}^{n}$.

The space-time FEM discretization is obtained by approximating the test and trial functions in each space-time element in the tessellation $\mathcal{K}^{n} \in \mathcal{T}_{h}^{n}$ with polynomial expansions that are assumed to be continuous within each space-time slab, but discontinuous across the interfaces of the space-time slabs, namely at times $t_{0}, t_{1}, \ldots, t_{N}$. 
The finite element space associated with the tessellation $\mathcal{T}_{h}^{n}$ is defined as

$$
V_{h}^{n}=\left\{u \in C\left(\mathcal{E}^{n}\right):\left.u\right|_{\mathcal{K}} \circ G_{\mathcal{K}}^{n} \in\left(\hat{\mathcal{P}}_{q}(-1,1) \otimes \hat{\mathcal{P}}_{r}(\hat{K})\right) \forall \mathcal{K} \in \mathcal{T}_{h}^{n}\right\},
$$

where $\hat{\mathcal{P}}_{q}(-1,1)$ and $\hat{\mathcal{P}}_{r}(\hat{K})$, respectively, represent $q$ th-order polynomials on $(-1,1)$ and $r$ th-order tensor product polynomials in the reference element $\hat{K}=(-1,1)^{d}$. Finally, define

$$
V_{h}=\left\{u \in L^{2}(\mathcal{E}):\left.u\right|_{\mathcal{E}_{n}} \in V_{h}^{n}, n=1,2, \ldots, N\right\} .
$$

Note that the functions in $V_{h}$ are allowed to be discontinuous at the nodes of the partition of the time interval. We will use the notation $u^{n, \pm}=\lim _{s \rightarrow 0^{ \pm}} u\left(t_{n}+s\right)$. Moreover, since $0 \notin I_{1}$, we specify $u^{0,-}=u_{0}(0)$.

The space-time dGcG-FEM method applied to problem (5)-(6) can be formulated as follows: Find $u_{h} \in V_{h}$ such that

$$
\begin{aligned}
& \sum_{n=1}^{N} \sum_{\mathcal{K} \in \mathcal{T}_{h}^{n}}\left[\left(\frac{\partial u_{h}}{\partial t}+\alpha u_{h}, v_{h}\right)_{\mathcal{K}}-\int_{\mathcal{K}}\left[\int_{\Omega} J(x, r) S\left(u_{h}(t-\tau(x, r), r)\right) d r\right] v_{h}(t, x) d x d t\right] \\
(8) \quad+ & \sum_{n=2}^{N}\left(\left[u_{h}\right]_{n-1}, v_{h}^{n-1,+}\right)+\left(u_{h}^{0,+}, v_{h}^{0,+}\right)=\left(u_{0}(0), v_{h}^{0,+}\right)
\end{aligned}
$$

holds for all $v_{h} \in V_{h}$ and where $u_{h}^{0,-}=u_{0}(0)$. Here $\left[u_{h}\right]_{n}=u_{h}^{n,+}-u_{h}^{n,-}$ denotes the jump of $u_{h}$ at $t_{n}$ and $(\cdot, \cdot)_{\mathcal{K}}$ is the $L^{2}(\mathcal{K})$-inner product on a space-time element. The jumps were added to the weak formulation to ensure weak continuity between time slabs, since the basis functions in dGcG-FEM discretizations are discontinuous at the space-time slab boundary.

Note that throughout this paper the FEM solution will be denoted by $u_{h}$, which should not be confused with the state of the system notation introduced in section 2. Moreover, it is important to remark that, for $u_{h} \in V_{h}$, the segments $u_{h t}, t>0$, are not necessarily continuous but piecewise continuous on $\left[-\tau_{\max }, 0\right]$. Denoting the space of piecewise continuous functions on $\left[-\tau_{\max }, 0\right]$ by $\hat{X}=P C\left(\left[-\tau_{\max }, 0\right] ; Y\right)$, we define the operator $\hat{G}: \hat{X} \rightarrow Y$ as

$$
\hat{G} \psi=\int_{\Omega} J(\cdot, r) S(\psi(-\tau(\cdot, r), r)) d r, \quad \psi \in \hat{X} .
$$

Then the nonlinear integral operator in (8) is equal to $\hat{G}\left(u_{h t}\right)$.

The weak formulation (8) can be transformed into an integrated-by-parts form, and since we added the jump term at each time level, it is possible to drop the summation over the space-time slabs. Moreover, after integration by parts, (8) can be decoupled into a sequence of local problems by choosing test functions that have support only in a single space-time slab $\mathcal{E}^{n}$. Hence we can solve the problem successively, i.e., using the known value $u_{h}\left(t_{n-1}^{-}\right)$from the previous space-time slab. The weak formulation for the dGcG-FEM discretization of the neural field equation is the following.

Find $u_{h} \in V_{h}^{n}$, such that for all $v_{h} \in V_{h}^{n}$ the variational equation is satisfied:

$$
\begin{aligned}
& \int_{\mathcal{K}^{n}}\left(-u_{h} \frac{\partial v_{h}}{\partial t}+\alpha u_{h} v_{h}\right) d x d t+\int_{K\left(t_{n}\right)} u_{h}^{n,-} v_{h}^{n,-} d x \\
& -\int_{\mathcal{K}^{n}} \hat{G}\left(u_{h t}\right)(x) v_{h} d x d t=\int_{K\left(t_{n-1}\right)} u_{h}^{n-1,-} v_{h}^{n-1,+} d x,
\end{aligned}
$$

Copyright $@$ by SIAM. Unauthorized reproduction of this article is prohibited. 


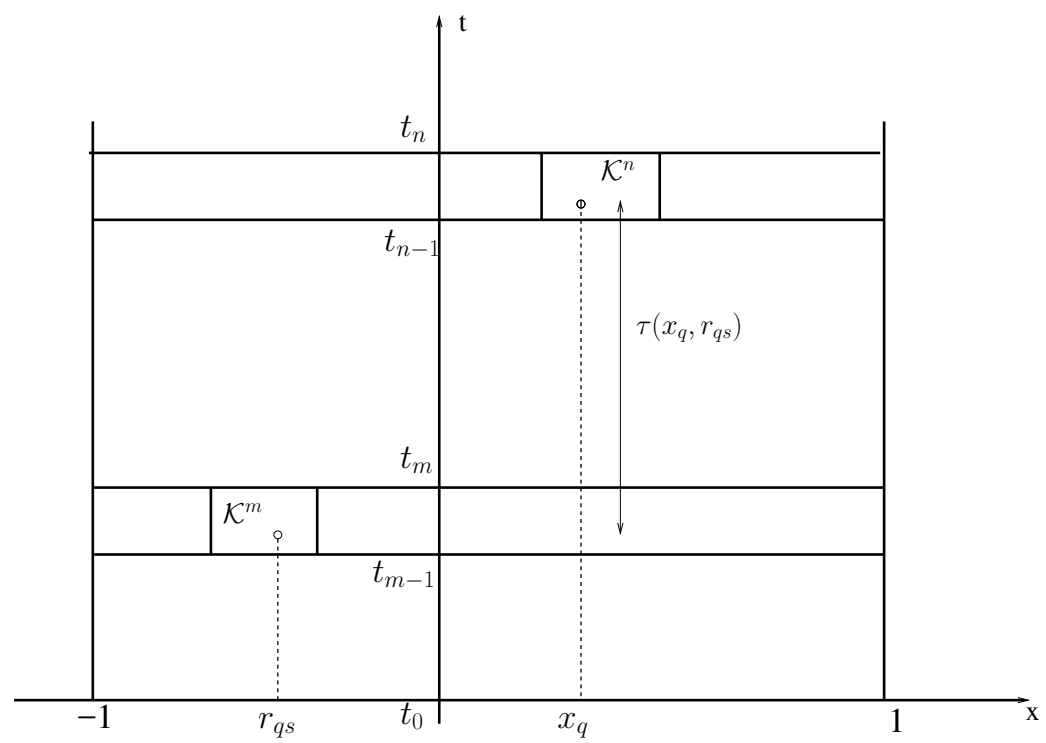

FIG. 2. The computational domain.

with $\mathcal{K}^{n} \in \mathcal{T}_{h}^{n}$ for $n=1, \ldots, N$.

Note here that the delay term may use values from space-time slabs where the solution was computed previously, but also from the current space-time slab, depending on the magnitude of the delay function compared to the time step. This problem will be discussed later in detail.

3.2. How to treat the delay term? In this section we discuss the dGcGFEM approximation of the delay term in the weak formulation (10). Introduce the approximation

$$
\left.u_{h}(t, x)\right|_{\mathcal{K}}=\sum_{m=1}^{N_{p}} \hat{u}_{m}^{\mathcal{K}} \psi_{m}^{\mathcal{K}}(t, x)
$$

into (10) and set the test function $\left.v_{h}(t, x)\right|_{\mathcal{K}}=\psi_{i}^{\mathcal{K}}(t, x), i \in\left\{1, \ldots, N_{p}\right\}$, with $N_{p}$ the number of degrees of freedom in element $\mathcal{K}$ and $\psi_{i}^{\mathcal{K}}$ standard Lagrange tensor product basis functions. The delay term becomes

$$
\begin{aligned}
& \int_{\mathcal{K}} \psi_{i}^{\mathcal{K}}(t, x)\left(\int_{\Omega} J(x, r) S\left(u_{h}(t-\tau(x, r), r)\right) d r\right) d x d t \\
& =\int_{\mathcal{K}} \psi_{i}^{\mathcal{K}}(t, x)\left(\sum_{L \in \overline{\mathcal{T}}_{h}} \int_{L} J(x, r) S\left(\sum_{m=1}^{N_{p}} \hat{u}_{m}^{L} \psi_{m}^{L}(t-\tau(x, r), r)\right) d r\right) d x d t .
\end{aligned}
$$

All integrals in the weak formulation are evaluated using Gaussian quadrature rules. Let us fix a quadrature point $\left(t_{q}, x_{q}\right) \in \mathcal{K}^{n}$ in a space-time element and let $\tau_{\max }=$ $\max _{(x, r) \in \bar{\Omega} \times \bar{\Omega}} \tau(x, r)$, as before. To compute the integral over a space element $L$ in (12), consider a space quadrature point $r_{q s} \in \Omega$, and distinguish three cases for the time delay $t_{q}-\tau\left(x_{q}, r_{q s}\right)$; see Figure 2 .

Case 1. If $-\tau_{\max } \leq t_{q}-\tau\left(x_{q}, r_{q s}\right) \leq 0$, then the solution at this time level is given by the initial solution, i.e., $u_{h}\left(t_{q}-\tau\left(x_{q}, r_{q s}\right), r_{q s}\right)=u_{0}\left(t_{q}-\tau\left(x_{q}, r_{q s}\right), r_{q s}\right)$. 
Case 2. When $t_{q}-\tau\left(x_{q}, r_{q s}\right) \geq t_{n-1}$, then the delay term (12) is implicit since we remain in the same space-time slab $\mathcal{E}^{n}$, where the solution is unknown. Hence, when the delay time is small enough compared to the time step, an additional Newton method needs to be incorporated for the solution of the nonlinear system.

If we introduce the finite element approximations for $u_{h}$ and $v_{h}$ also into the other terms in the weak formulation (10), then we obtain for all $\mathcal{K} \in \mathcal{T}_{h}^{n}$

$$
\begin{aligned}
& \sum_{j=1}^{N_{p}}\left\{\hat{u}_{j}^{\mathcal{K}} \int_{\mathcal{K}}\left(-\psi_{j}^{\mathcal{K}}(t, x) \frac{\partial}{\partial t} \psi_{i}^{\mathcal{K}}(t, x)+\alpha \psi_{j}^{\mathcal{K}}(t, x) \psi_{i}^{\mathcal{K}}(t, x)\right) d x d t\right. \\
& \left.\quad+\hat{u}_{j}^{\mathcal{K}} \int_{K\left(t_{n}^{-}\right)} \psi_{j}^{\mathcal{K}}\left(t_{n}^{-}, x\right) \psi_{i}^{\mathcal{K}}\left(t_{n}^{-}, x\right) d x\right\} \\
& -\sum_{L \in \overline{\mathcal{T}}_{h}} \int_{\mathcal{K}} \psi_{i}^{\mathcal{K}}(t, x)\left[\int_{L} J(x, r) S\left(\sum_{m=1}^{N_{p}} \hat{u}_{m}^{L} \psi_{m}^{L}(t-\tau(x, r), r)\right) d r\right] d x d t \\
& =\sum_{j=1}^{N_{p}} \hat{u}_{j}^{\mathcal{K}, n-1} \int_{K\left(t_{n-1}^{+}\right)} \psi_{j}^{\mathcal{K}}\left(t_{n-1}^{-}, x\right) \psi_{i}^{\mathcal{K}}\left(t_{n-1}^{+}, x\right) d x,
\end{aligned}
$$

where $\hat{u}_{j}^{\mathcal{K}, n-1}$ are the coefficients of space-time element $\mathcal{K}$ in the space-time slab $\mathcal{E}^{n-1}$.

Case 3. When $0 \leq t_{q}-\tau\left(x_{q}, r_{q s}\right)<t_{n-1}$, then the delay term is explicit since we go back to a previous space-time slab, where the FEM solution is already computed.

4. Error analysis. In this section we give an a priori error analysis for the space-time dGcG method (13). In the error analysis we will use a slightly modified version of the temporal interpolation functions defined in [12, Proposition 4.1]. First, define the space

$$
S_{k}=\left\{w:[0, T] \rightarrow Y:\left.w\right|_{I_{n}}=\sum_{j=0}^{q} \varphi_{j} t^{j}, \varphi_{j} \in Y, \forall n \geq 1\right\},
$$

with $I_{n}=\left(t_{n-1}, t_{n}\right]$ and $\left|I_{n}\right|=k_{n}$. Note that these functions are allowed to be discontinuous at the nodes of the partition of the time interval, but continuous from the left in each subinterval $I_{n}$, i.e., $w\left(t_{n}\right)=\lim _{t \rightarrow t_{n}^{-}} w(t)$. For the restriction of the functions in $S_{k}$ to $I_{n}$, we use the notation $S_{k}^{n}$. Define the temporal polynomial interpolant

$$
T_{k}: C([0, T], Y) \rightarrow S_{k}
$$

as follows; see also [12].

Proposition 1. Let $\tilde{u}=T_{k} u \in S_{k}$ be the time-interpolant of $u \in C([0, T], Y) \cap$ $H^{q+1}([0, T], Y), q \geq 0$, with the following properties:

$$
\begin{gathered}
\tilde{u}\left(t_{n-1}\right)=u\left(t_{n-1}\right) \quad \text { for } n \geq 1, \\
\int_{I_{t}}(\tilde{u}(s)-u(s)) s^{l} d s=0 \quad \text { for } l=0, \ldots, q-1, t \in I_{n}, I_{t}=\left(t_{n-1}, t\right], n \geq 1 .
\end{gathered}
$$

Copyright $@$ by SIAM. Unauthorized reproduction of this article is prohibited. 
The interpolation error then can be estimated as

$$
\|\tilde{u}(s)-u(s)\| \leq C_{I} k_{t}^{q+1 / 2}\left(\int_{I_{t}}\left\|\partial_{s}^{q+1} u(s, \cdot)\right\|^{2} d s\right)^{1 / 2} \text { for } s \in I_{t},
$$

where $\partial_{s}^{q+1}$ denotes the $(q+1)$ th-order derivative w.r.t. time, $k_{t}=\left|I_{t}\right|$, and the norm $\|\cdot\|=\|\cdot\|_{L^{2}(\Omega)}$ hereafter.

Observe that $\tilde{u}$ interpolates exactly at the nodes and the interpolation error is orthogonal to polynomials of degree at most $q-1$. For constant polynomials $(q=0)$ condition (17) is not used.

Next, define the spatial interpolant. Let $W_{h}$ be the space of tensor product polynomials of degree up to $r \geq 0$ on each space element $K_{j}$, i.e.,

$$
W_{h}=\left\{v \in C(\Omega):\left.v\right|_{K} \circ G_{K}^{n} \in \hat{\mathcal{P}}_{r}(\hat{K}) \forall K \in \overline{\mathcal{T}}_{h}\right\},
$$

where $G_{K}^{n}$ denotes the mapping from the reference element $\hat{K}=(-1,1)^{d}$ to the element $K \in \overline{\mathcal{T}}_{h}$ in physical space. Let

$$
P_{h}: Y \rightarrow W_{h}
$$

be the $L^{2}$-projection to the (spatial) finite element space, defined as $\left(P_{h} v, w_{h}\right)=$ $\left(v, w_{h}\right)$ for all $w_{h} \in W_{h}$. We use the standard interpolation estimate in space (see, e.g., $[2],[3])$

$$
\left\|v-P_{h} v\right\| \leq C h^{r+1}\|v\|_{r+1} \quad \forall v \in Y \cap H^{r+1}(\Omega),
$$

where $\|\cdot\|_{r+1}=\|\cdot\|_{H^{r+1}(\Omega)}, h$ denotes the maximal space element diameter as before, and the constant $C$ is independent of $h$ and $v$.

In the error analysis we also need the interpolation of the initial segment of the solution. Let the given initial function be $u_{0} \in X \cap H^{q+1}\left(\left[-\tau_{\max }, 0\right] ; H^{r+1}(\Omega)\right)$ for some $q, r \geq 0$. Use a partition of the interval $\left[-\tau_{\max }, 0\right]$ into $M$ subintervals $J_{i}$ of length $k_{i}$, respectively. On each $J_{i}$ we use the same temporal interpolation $\tilde{u}_{0}=T_{k} u_{0}$ of $u_{0}$, as introduced in Proposition 1. Then for all $s \in J_{i}$ we have

$$
\begin{aligned}
\| u_{0}(s) & -P_{h} T_{k} u_{0}(s)\|=\| u_{0}(s)-P_{h} \tilde{u}_{0}(s) \| \\
& \leq\left\|u_{0}(s)-P_{h} u_{0}(s)\right\|+\left\|P_{h}\right\|\left\|u_{0}(s)-T_{k} u_{0}(s)\right\| \\
& \leq C h^{r+1}\left\|u_{0}(s)\right\|_{r+1}+C_{I} k_{i}^{q+1 / 2}\left(\int_{J_{i}}\left\|\partial_{s}^{q+1} u_{0}(s, \cdot)\right\|^{2} d s\right)^{1 / 2}
\end{aligned}
$$

where we use that the operator norm of the Lagrange interpolation $P_{h}$ is bounded; see [2], [4].

We will also need an estimate of the integral of the interpolation error on the partition of the initial segment. There exists $C>0$, a generic constant (independent of the solution and mesh size), such that

$$
\begin{aligned}
& \int_{J_{i}}\left\|u_{0}(s)-P_{h} T_{k} u_{0}(s)\right\|^{2} d s \leq C \mathcal{B}\left(u_{0}, J_{i}\right) \\
& \quad:=C\left(h^{2 r+2} k_{i}\left\|u_{0}\right\|_{r+1, J_{i}}^{2}+k_{i}^{2 q+2} \int_{J_{i}}\left\|\partial_{s}^{q+1} u_{0}(s, \cdot)\right\|^{2} d s\right),
\end{aligned}
$$

Copyright $@$ by SIAM. Unauthorized reproduction of this article is prohibited. 
where we denote the norm

$$
\|\varphi\|_{r, I}=\sup _{t \in I}\|\varphi(t)\|_{r}
$$

Next, we state the main result of the a priori error analysis of the dGcG discretization (10) for the neural field equations.

Theorem 2. Let $u \in C^{1}([0, T) ; Y) \cap H^{q+1}\left([0, T] ; H^{r+1}(\Omega)\right)$ be the solution of (4) for some $q, r \geq 0$ and with initial state $u_{0} \in X \cap H^{q+1}\left(\left[-\tau_{\max }, 0\right] ; H^{r+1}(\Omega)\right)$, and let $u_{h} \in V_{h}^{n}$ be the solution of (10). Then

$$
\begin{aligned}
\left\|u_{h}\left(t_{N}\right)-u\left(t_{N}\right)\right\|^{2} \leq C & \left(\sum_{i=1}^{M} m(i) \mathcal{B}\left(u_{0}, J_{i}\right)+\sum_{n=1}^{N} m(n) k_{n}^{2 q+2} \int_{I_{n}}\left\|\partial_{t}^{q+1} u(t, \cdot)\right\|^{2} d t\right. \\
& \left.+h^{2 r+2} \sum_{n=0}^{N}\left\|u\left(t_{n}\right)\right\|_{r+1}^{2}+\sum_{n=1}^{N} h^{2 r+2} m(n) k_{n}\|u\|_{r+1, I_{n}}^{2}\right)
\end{aligned}
$$

holds for $t_{N} \geq 0, N$ the number of time slabs, where $C$ is a positive constant independent of the time step $k_{n}=t_{n}-t_{n-1}$ and the maximal space element diameter $h$. Here $m(n) \leq N-1,1 \leq n \leq N$, is the multiplicity of how many times we visited the interval $I_{n}$ due to the delay term.

Proof. Let us decompose the error of the numerical discretization into the sum

$$
\begin{aligned}
\left(u_{h}-u\right)(t, x) & =\left[u_{h}(t, x)-P_{h} \tilde{u}(t)(x)\right]+\left[P_{h} \tilde{u}(t)(x)-u(t, x)\right] \\
& =\theta(t, x)+\rho(t, x) \quad \text { for } t>0,
\end{aligned}
$$

with $\theta$ the discretization error and $\rho$ the interpolation error. When $t \in\left[-\tau_{\max }, 0\right]$, we only have the interpolation error of the given initial solution $u_{0}$, that is, $\theta(t, x)=0$ and $\rho(t, x)=P_{h} \tilde{u}_{0}(t)(x)-u_{0}(t, x)$. From here on, we suppress the spatial dependence where it is clear from the context. Since $\tilde{u}$ interpolates exactly at the nodes $t=t_{n-1}$, we have that

$$
\begin{aligned}
\left\|\rho\left(t_{n-1}\right)\right\| & =\left\|P_{h} T_{k} u\left(t_{n-1}\right)-u\left(t_{n-1}\right)\right\| \\
& =\left\|P_{h} u\left(t_{n-1}\right)-u\left(t_{n-1}\right)\right\| \leq C h^{r+1}\left\|u\left(t_{n-1}\right)\right\|_{r+1}
\end{aligned}
$$

holds for all $n \geq 1$. Here the constant $C$ is independent of $h$; see, e.g., [2]. When we are in the interior of a time interval $I_{j}$, we decompose $\rho$ to be able to use the bound on the interpolation error in time and space, respectively, as in (21):

$$
\begin{aligned}
\|\rho(t)\| & =\left\|P_{h} T_{k} u(t)-u(t)\right\| \\
& \leq C\left(h^{r+1}\|u(t)\|_{r+1}+k_{j}^{q+1 / 2}\left(\int_{I_{j}}\left\|\partial_{s}^{q+1} u(s, \cdot)\right\|^{2} d s\right)^{1 / 2}\right)
\end{aligned}
$$

for any $t \in I_{j}$ and $j=1, \ldots, N$. It is, therefore, sufficient to bound $\theta^{N}=\theta\left(t_{N}\right)$. Since both $u_{h}$ and $u$ satisfy the weak formulation (10) with $G$ and $\hat{G}$, respectively, we obtain that for all $v \in V_{h}^{n}$

$$
\begin{aligned}
\int_{I_{n}} & \left(\frac{\partial}{\partial t} \theta(t)+\alpha \theta(t), v(t)\right) d t+\left([\theta]_{n-1}, v^{n-1,+}\right) \\
= & \int_{I_{n}}\left(-\frac{\partial}{\partial t} \rho(t)-\alpha \rho(t)+\hat{G}\left(u_{h t}\right)-G\left(u_{t}\right), v(t)\right) d t-\left([\rho]_{n-1}, v^{n-1,+}\right) .
\end{aligned}
$$

Copyright (c) by SIAM. Unauthorized reproduction of this article is prohibited. 
The variational equation (27) holds for any partition of the time interval $I_{n}$, and hence the following equation is also valid for any $t \in\left(t_{n-1}, t_{n}\right]$ :

$$
\begin{aligned}
\int_{I_{t}} & \left(\frac{\partial}{\partial s} \theta(s)+\alpha \theta(s), v(s)\right) d s+\left([\theta]_{n-1}, v^{n-1,+}\right) \\
& =\int_{I_{t}}\left(-\frac{\partial}{\partial s} \rho(s)-\alpha \rho(s)+\hat{G}\left(u_{h_{s}}\right)-G\left(u_{s}\right), v(s)\right) d s-\left([\rho]_{n-1}, v^{n-1,+}\right),
\end{aligned}
$$

where $I_{t}=\left(t_{n-1}, t\right]$. Using the assumptions on the interpolant, some terms in (28) will cancel, i.e., for all $t \in I_{n}$

$$
\begin{aligned}
\int_{I_{t}} & \left(\frac{\partial}{\partial s} \rho(s), v(s)\right) d s+\left(\rho\left(t_{n-1}^{+}\right)-\rho\left(t_{n-1}^{-}\right), v^{n-1,+}\right) \\
& =-\int_{I_{t}}\left(\rho(s), \frac{\partial}{\partial s} v(s)\right) d s+\left.(\rho(s), v(s))\right|_{t=t_{n-1}^{+}} ^{t}+\left(\rho^{n-1,+}-\rho^{n-1,-}, v^{n-1,+}\right) \\
& =-\int_{I_{t}}\left(\rho(s), \frac{\partial}{\partial s} v(s)\right) d s+(\rho(t), v(t))-\left(\rho^{n-1,-}, v^{n-1,+}\right) \\
(29) \quad & (\rho(t), v(t))-\left(\rho^{n-1,-}, v^{n-1,+}\right) .
\end{aligned}
$$

Let $v=2 \theta \in S_{k}^{n}$ in (28). Then for each $I_{n}$ and $t \in I_{n}$ the following holds:

$$
\begin{aligned}
& \int_{I_{t}} 2\left(\frac{\partial}{\partial s} \theta(s)+\alpha \theta(s), \theta(s)\right) d s+2\left([\theta]_{n-1}, \theta^{n-1,+}\right) \\
& \quad \stackrel{(30)}{\quad=} \int_{I_{t}} 2\left(-\alpha \rho(s)+\hat{G}\left(u_{h_{s}}\right)-G\left(u_{s}\right), \theta(s)\right) d s-2(\rho(t), \theta(t))+2\left(\rho^{n-1,-}, \theta^{n-1,+}\right) .
\end{aligned}
$$

This may be further written as

$$
\begin{aligned}
& \int_{I_{t}}\left[\frac{d}{d s}\|\theta(s)\|^{2}+2 \alpha\|\theta(s)\|^{2}\right] d s+2\left\|\theta^{n-1,+}\right\|^{2}=2\left(\theta^{n-1,-}, \theta^{n-1,+}\right) \\
& \quad+\int_{I_{t}} 2\left(-\alpha \rho(s)+\hat{G}\left(u_{h_{s}}\right)-G\left(u_{s}\right), \theta(s)\right) d s-2(\rho(t), \theta(t))+2\left(\rho^{n-1,-}, \theta^{n-1,+}\right) .
\end{aligned}
$$

Using the Schwarz inequality and the inequality $2 a b \leq \epsilon^{2} a^{2}+\frac{1}{\epsilon^{2}} b^{2}$, we obtain

$$
\begin{aligned}
\left(1-\epsilon^{2}\right)\|\theta(t)\|^{2} \leq & -2 \alpha \int_{I_{t}}\|\theta(s)\|^{2} d s+2\left\|\theta^{n-1,-}\right\|^{2} \\
& +\alpha \int_{I_{t}}\left(\|\rho(s)\|^{2}+\|\theta(s)\|^{2}\right) d s+\frac{1}{\epsilon^{2}}\|\rho(t)\|^{2} \\
& +2 \int_{I_{t}}\left(\hat{G}\left(u_{h_{s}}\right)-G\left(u_{s}\right), \theta(s)\right) d s+2\left\|\rho^{n-1,-}\right\|^{2} .
\end{aligned}
$$

Since the nonlinearity $S$ is Lipschitz continuous with some Lipschitz constant $C_{S}$, we 
can estimate the nonlinear term as

$$
\begin{aligned}
& 2 \int_{I_{t}}\left(\hat{G}\left(u_{h s}\right)-G\left(u_{s}\right), \theta(s)\right) d s \\
& =2 \int_{I_{t}} \int_{\Omega}\left[\int_{\Omega} J(x, r)\left[S\left(u_{h}(s-\tau(x, r), r)\right)-S(u(s-\tau(x, r), r))\right] d r\right] \theta(s, x) d x d s \\
& (33) \\
& \leq 2 C_{S} \int_{I_{t}} \int_{\Omega}\left[\int_{\Omega}|J(x, r)|(|\theta(s-\tau(x, r), r)|+|\rho(s-\tau(x, r), r)|) d r\right] \theta(s, x) d x d s .
\end{aligned}
$$

Let us estimate the first term on the right-hand side of (33) as

$$
\begin{aligned}
T_{1}:= & \int_{I_{t}} \int_{\Omega}\left[\int_{\Omega}|J(x, r)||\theta(s-\tau(x, r), r)| d r\right] \theta(s, x) d x d s \\
& \leq \int_{I_{t}}\left(\int_{\Omega}\left(\int_{\Omega}|J(x, r)||\theta(s-\tau(x, r), r)| d r\right)^{2} d x\right)^{1 / 2}\left(\int_{\Omega} \theta^{2}(s, x) d x\right)^{1 / 2} d s \\
& \leq \int_{I_{t}}\left(|\Omega| \int_{\Omega} \int_{\Omega} J^{2}(x, r) \theta^{2}(s-\tau(x, r), r) d r d x\right)^{1 / 2}\left(\int_{\Omega} \theta^{2}(s, x) d x\right)^{1 / 2} d s \\
(34) & \leq\left(|\Omega| \int_{I_{t}} \int_{\Omega} \int_{\Omega} J^{2}(x, r) \theta^{2}(s-\tau(x, r), r) d r d x d s\right)^{1 / 2}\left(\int_{I_{t}} \int_{\Omega} \theta^{2}(s, x) d x d s\right)^{1 / 2},
\end{aligned}
$$

where we used the Schwarz inequality in each estimation step and $|\Omega|=\operatorname{vol}(\Omega)$. Next, since $0<\tau(x, r) \leq \tau_{\max }$ and $J(x, r) \leq\|J\|_{C}$, for all $(x, r) \in \bar{\Omega} \times \bar{\Omega}$, the following estimate is valid:

$$
\begin{aligned}
\int_{I_{t}} \int_{\Omega} \int_{\Omega} J^{2}(x, r) \theta^{2}(s-\tau(x, r), r) d r d x d s \\
\leq\|J\|_{C}^{2} \int_{I_{t}} \int_{\Omega} \int_{\Omega} \theta^{2}(s-\tau(x, r), r) d r d x d s \\
\leq\|J\|_{C}^{2}|\Omega| \int_{t_{n-1}-\tau_{\max }}^{t} \int_{\Omega} \theta^{2}(s, r) d r d s
\end{aligned}
$$

Hence we can further estimate (34) as

$$
T_{1} \leq\|J\|_{C}|\Omega|\left(\int_{t_{n-1}-\tau_{\max }}^{t}\|\theta(s)\|^{2} d s\right)^{1 / 2}\left(\int_{I_{t}}\|\theta(s)\|^{2} d s\right)^{1 / 2}
$$

$\leq\|J\|_{C}|\Omega| \int_{t_{n-1}-\tau_{\max }}^{t}\|\theta(s)\|^{2} d s=\|J\|_{C}|\Omega|\left(\int_{t_{n-1}-\tau_{\max }}^{t_{n-1}}\|\theta(s)\|^{2} d s+\int_{I_{t}}\|\theta(s)\|^{2} d s\right)$.

Copyright (C) by SIAM. Unauthorized reproduction of this article is prohibited. 
Similarly as in (34) and (35), for the last term in (33) we obtain

$$
\begin{aligned}
T_{2}:= & 2 \int_{I_{t}} \int_{\Omega}\left[\int_{\Omega}|J(x, r) \| \rho(s-\tau(x, r), r)| d r\right] \theta(s, x) d x d s \\
& \leq 2\|J\|_{C}|\Omega|\left(\int_{t_{n-1}-\tau_{\max }}^{t}\|\rho(s)\|^{2} d s\right)^{1 / 2}\left(\int_{I_{t}}\|\theta(s)\|^{2} d s\right)^{1 / 2} \\
& \leq\|J\|_{C}^{2}|\Omega|^{2} \int_{t_{n-1}-\tau_{\max }}^{t_{n}}\|\rho(s)\|^{2} d s+\int_{I_{t}}\|\theta(s)\|^{2} d s .
\end{aligned}
$$

After introducing the above estimates into (32) we obtain that for all $t \in I_{n}$,

$$
\begin{aligned}
\left(1-\epsilon^{2}\right)\|\theta(t)\|^{2} \leq & \left(C_{S}-\alpha+2 C_{S}\|J\|_{C}|\Omega|\right) \int_{I_{t}}\|\theta(s)\|^{2} d s+2\left\|\theta^{n-1}\right\|^{2} \\
& +\alpha \int_{I_{n}}\|\rho(s)\|^{2} d s+2 C_{S}\|J\|_{C}|\Omega| \int_{t_{n-1}-\tau_{\max }}^{t_{n-1}}\|\theta(s)\|^{2} d s \\
& +C_{S}\|J\|_{C}^{2}|\Omega|^{2} \int_{t_{n-1}-\tau_{\max }}^{t_{n}}\|\rho(s)\|^{2} d s+\frac{1}{\epsilon^{2}}\|\rho(t)\|^{2}+2\left\|\rho^{n-1}\right\|^{2}
\end{aligned}
$$

is valid for all $n \geq 1$. Divide by $1-\epsilon^{2}$, where $0<\epsilon<1$, and denote

$$
\begin{aligned}
& \beta=\frac{\left|C_{S}-\alpha+2 C_{S}\|J\|_{C}\right| \Omega||}{1-\epsilon^{2}}>0, \\
& \omega_{n}(t)=\gamma_{n}+\frac{1}{\epsilon^{2}\left(1-\epsilon^{2}\right)}\|\rho(t)\|^{2}, \\
& \gamma_{n}=\frac{1}{1-\epsilon^{2}}\left(2\left\|\theta^{n-1}\right\|^{2}+\alpha \int_{I_{n}}\|\rho(s)\|^{2} d s+2 C_{S}\|J\|_{C}|\Omega| \int_{t_{n-1}-\tau_{\max }}^{t_{n-1}}\|\theta(s)\|^{2} d s\right. \\
& \left.\quad+C_{S}\|J\|_{C}^{2}|\Omega|^{2} \int_{t_{n-1}-\tau_{\max }}^{t_{n}}\|\rho(s)\|^{2} d s+2\left\|\rho^{n-1}\right\|^{2}\right), \quad n \geq 1 .
\end{aligned}
$$

Then, inequality (38) can be written as

$$
\eta(t) \leq \omega_{n}(t)+\beta \int_{I_{t}} \eta(s) d s, t \in I_{n}
$$

where $\eta(t)=\|\theta(t)\|^{2}$. Apply Grönwall's inequality to (39) to obtain

$$
\eta(t) \leq \omega_{n}(t)+\beta \int_{I_{t}} \omega_{n}(s) e^{\beta(t-s)} d s, t \in I_{n} .
$$

When $t=t_{n}$,

$$
\eta\left(t_{n}\right) \leq \omega_{n}\left(t_{n}\right)+\beta \int_{I_{n}} \omega_{n}(s) e^{\beta\left(t_{n}-s\right)} d s
$$

where

$$
\omega_{n}\left(t_{n}\right)=\gamma_{n}+\frac{1}{\epsilon^{2}\left(1-\epsilon^{2}\right)}\left\|\rho\left(t_{n}\right)\right\|^{2}, \quad n \geq 1 .
$$

Copyright $@$ by SIAM. Unauthorized reproduction of this article is prohibited. 
Note that the only time-dependent term in $\omega_{n}(t)$ is $\|\rho(t)\|^{2}$. Hence, the integral term in (41) can be estimated as

$$
\begin{aligned}
\int_{I_{n}} \omega_{n}(s) e^{\beta\left(t_{n}-s\right)} d s & =\int_{I_{n}}\left(\gamma_{n}+\frac{1}{\epsilon^{2}\left(1-\epsilon^{2}\right)}\|\rho(s)\|^{2}\right) e^{\beta\left(t_{n}-s\right)} d s \\
& \leq e^{\beta k_{n}}\left(k_{n} \gamma_{n}+\frac{1}{\epsilon^{2}\left(1-\epsilon^{2}\right)} \int_{I_{n}}\|\rho(s)\|^{2} d s\right) .
\end{aligned}
$$

Therefore, we obtain for $n \geq 1$ that

$$
\eta\left(t_{n}\right) \leq\left(1+\beta k_{n} e^{\beta k_{n}}\right) \gamma_{n}+\frac{\beta e^{\beta k_{n}}}{\epsilon^{2}\left(1-\epsilon^{2}\right)} \int_{I_{n}}\|\rho(s)\|^{2} d s+\frac{1}{\epsilon^{2}\left(1-\epsilon^{2}\right)}\left\|\rho^{n}\right\|^{2} .
$$

Let us recall that

$$
\begin{gathered}
\gamma_{n}=\frac{1}{1-\epsilon^{2}}\left(2 \eta\left(t_{n-1}\right)+2 C_{S}\|J\|_{C}|\Omega| \int_{t_{n-1}-\tau_{\max }}^{t_{n-1}} \eta(s) d s+\alpha \int_{I_{n}}\|\rho(s)\|^{2} d s\right. \\
\left.+C_{S}\|J\|_{C}^{2}|\Omega|^{2} \int_{t_{n-1}-\tau_{\max }}^{t_{n}}\|\rho(s)\|^{2} d s+2\left\|\rho^{n-1}\right\|^{2}\right)
\end{gathered}
$$

and observe that the right-hand side of (44) can be estimated by the bound of the interpolation error and the bound of the integral of $\eta(t)$ over earlier time intervals, i.e., for $t \leq t_{n-1}$. Hence we can write

$$
\begin{aligned}
\eta\left(t_{n}\right) & \leq C_{1} \eta\left(t_{n-1}\right)+C_{2} \int_{t_{n-1}-\tau_{\max }}^{t_{n-1}} \eta(s) d s \\
& +C_{3} \int_{t_{n-1}-\tau_{\max }}^{t_{n}}\|\rho(s)\|^{2} d s+C_{4}\left\|\rho^{n-1}\right\|^{2}+\frac{1}{\epsilon^{2}\left(1-\epsilon^{2}\right)}\left\|\rho^{n}\right\|^{2},
\end{aligned}
$$

where $C_{i}, i=1, \ldots, 4$, depend on the parameters $\alpha, \beta, \epsilon,\|J\|_{C},|\Omega|$ and $k_{n}$, such that $C_{i}=O(1)$ as $k_{n} \rightarrow 0$.

By integrating (40) we obtain the following general formula:

$$
\begin{aligned}
\int_{t_{n-1}-\tau_{\max }}^{t_{n-1}} \eta(s) d s & \leq \sum_{j=m(n)}^{n-1} \int_{I_{j}} \eta(s) d s \\
& \leq \sum_{j=m(n)}^{n-1} \int_{I_{j}}\left(\omega_{j}(s)+\beta \int_{I_{s}} \omega_{j}(\tau) e^{\beta(s-\tau)} d \tau\right) d s \\
& \leq \sum_{j=m(n)}^{n-1} \int_{I_{j}}\left(\omega_{j}(s)+\beta \int_{I_{j}} \omega_{j}(\tau) e^{\beta\left(t_{j}-\tau\right)} d \tau\right) d s \\
& \leq \sum_{j=m(n)}^{n-1}\left(1+k_{j} \beta e^{\beta k_{j}}\right) \int_{I_{j}} \omega_{j}(s) d s \\
& \leq \sum_{j=m(n)}^{n-1}\left(1+k_{j} \beta e^{\beta k_{j}}\right)\left(k_{j} \gamma_{j}+\frac{1}{\epsilon^{2}\left(1-\epsilon^{2}\right)} \int_{I_{j}}\|\rho(s)\|^{2} d s\right)
\end{aligned}
$$

Copyright $@$ by SIAM. Unauthorized reproduction of this article is prohibited. 
where we used that $\eta(s)=\|\theta(s)\|^{2}=0$ for $s \in\left[-\tau_{\max }, 0\right]$ and (43) in the last inequality. Here $m=m(n) \leq n-1$ is the index of the interval $I_{m}$ for which $t_{n-1}-$ $\tau_{\max } \in I_{m}$.

As we can see, the integral of $\eta(s)$ can be bounded by the integral of $\|\rho(s)\|$, and hence in (46) we have

$$
\begin{aligned}
\eta\left(t_{n}\right) & \leq C \sum_{j=m(n)}^{n-1}\left[\left(1+k_{j} \beta e^{\beta k_{j}}\right) k_{j} \gamma_{j}+\left(\frac{1+k_{j} \beta e^{\beta k_{j}}}{\epsilon^{2}\left(1-\epsilon^{2}\right)}+1\right) \int_{I_{j}}\|\rho(s)\|^{2} d s\right] \\
& +C_{3} \int_{I_{n}}\|\rho(s)\|^{2} d s+C_{4}\left\|\rho^{n-1}\right\|^{2}+\frac{1}{\epsilon^{2}\left(1-\epsilon^{2}\right)}\left\|\rho^{n}\right\|^{2}+C_{1} \eta\left(t_{n-1}\right) .
\end{aligned}
$$

We can use (26) to bound the integral of $\|\rho(s)\|$ as follows:

$$
\int_{I_{j}}\|\rho(s)\|^{2} d s=C\left[h^{2 r+2} k_{j}\|u\|_{r+1, I_{j}}^{2}+k_{j}^{2 q+2} \int_{I_{j}}\left\|\partial_{s}^{q+1} u(s, \cdot)\right\|^{2} d s\right] .
$$

For $n=1$, combining (46) with (21), (22), (25), (26) and (49) and using that $\eta(0)=0$, we find that there exists a generic constant $C$, independent of the time step $k_{1}$ and the spatial mesh size $h$, such that

$$
\begin{aligned}
\eta\left(t_{1}\right) & \leq C_{3} \int_{-\tau_{\max }}^{t_{1}}\|\rho(s)\|^{2}+C_{4}\left\|\rho^{0}\right\|^{2}+\frac{1}{\epsilon^{2}\left(1-\epsilon^{2}\right)}\left\|\rho^{1}\right\|^{2} \\
& \leq C\left[\sum_{i=1}^{M} \mathcal{B}\left(u_{0}, J_{i}\right)+h^{2 r+2}\left(\|u(0)\|_{r+1}^{2}+\left\|u\left(t_{1}\right)\right\|_{r+1}^{2}\right)\right. \\
& \left.+h^{2 r+2} k_{1}\|u\|_{r+1, I_{1}}^{2}+k_{1}^{2 q+2} \int_{I_{1}}\left\|\partial_{s}^{q+1} u(s, \cdot)\right\|^{2} d s\right] .
\end{aligned}
$$

For $n=2$, using again (46) and then (25), (26), (47) and (50), we find that there is a constant $C$, such that

$$
\begin{aligned}
\eta\left(t_{2}\right) & \leq C_{1} \eta\left(t_{1}\right)+C_{2} \int_{t_{1}-\tau_{\max }}^{t_{1}} \eta(s) d s+C_{3} \int_{t_{1}-\tau_{\max }}^{t_{2}}\|\rho(s)\|^{2} \\
& +C_{4}\left\|\rho^{1}\right\|^{2}+\frac{1}{\epsilon^{2}\left(1-\epsilon^{2}\right)}\left\|\rho^{2}\right\|^{2} \\
& \leq C\left[\sum_{i=1}^{M} m(i) \mathcal{B}\left(u_{0}, J_{i}\right)+h^{2 r+2}\left(\|u(0)\|_{r+1}^{2}+\left\|u\left(t_{1}\right)\right\|_{r+1}^{2}+\left\|u\left(t_{2}\right)\right\|_{r+1}^{2}\right)\right. \\
& \left.+h^{2 r+2} \sum_{j=1}^{2} m(j) k_{j}\|u\|_{r+1, I_{j}}^{2}+\sum_{j=1}^{2} m(j) k_{j}^{2 q+2} \int_{I_{j}}\left\|\partial_{s}^{q+1} u(s, \cdot)\right\|^{2} d s\right],
\end{aligned}
$$

where $m(i)$ and $m(j)$ are the multiplicity of how many times we visited the interval $J_{i}$ and $I_{j}$, respectively, in the integral of $\|\rho(s)\|$ over the delay interval. If $\tau_{\max }$ is large compared to the time step, then $m(i)$ is consequently also larger.

We can repeat this procedure for the subsequent time intervals, which completes the proof of the theorem.

Copyright $@$ by SIAM. Unauthorized reproduction of this article is prohibited. 
5. Numerical examples. In this section we discuss the complexity of the algorithm and present some applications of the dGcG-FEM discretization to neural field equations in one and two space dimensions.

The dGcG-method to solve neural field equations is essentially the same as other space-time methods for ordinary and partial differential equations. The main difference is the computation of the integral part that involves the history. It is well known that the complexity of numerical algorithms to solve integro-differential equations can be very high, especially in cases of several space dimensions. Neural field equations often have weight kernels that model the interactions of populations of neurons having an arbitrary spatial distribution. Depending on the kernel size, the localized nature of the problem then can be lost and the matrices arising from the numerical discretization will not be sparse. In that case, the linear system can still be solved efficiently using a fast multipole method, but this is not considered in the present study. The computational cost further increases when transmission time delays are considered.

In our dGcG method in each space-time slab we solve a nonlinear or linear system of equations of the size of the total number of degrees of freedom in the slab. The linearity, or nonlinearity, depends on the magnitude of the delay function compared to the time step, as discussed in section 3.2. When we solve the linear system, the integral part containing the delay is a source term, and it will contribute to the righthand side of the linear system. We need to compute this delay term each time step, which requires the evaluation of the delay integral for a number of old time levels. The cost of evaluating the delay integral per element is approximately the cost of computing the residual for one time step, times the number of delay time steps for that element. Depending on how large this delay is, this can computationally be expensive.

5.1. Space and time accuracy. To verify our results on the error analysis, we considered two examples: a DDE with one constant delay and an integro-differential equation without delay.

First, consider a DDE of the form

$$
\dot{u}(t)=f(u(t), u(t-\tau)), \quad t>0,
$$

with initial function $u(s)=u_{0}(s), s \in[-\tau, 0], \tau>0$ constant delay, and $f: \mathbb{R}^{2} \rightarrow \mathbb{R}$ linear, given by $f(u(t), u(t-\tau))=-\alpha u(t)+u(t-\tau)$. The numerical integration of these types of equations is very sensitive to jump discontinuities in the solution or in its derivatives. Such discontinuity points are referred to in the literature as breaking points [1]. The best procedure to guarantee the required accuracy is to include these breaking points in the set of mesh points. Figure 3 illustrates the solution of (52) with $u_{0}(s)=-s, s \in[-\tau, 0], \tau=2$, when $\alpha=1$, for which we know that it converges to a nonzero steady state. We use the dG(1) method, using linear basis functions, set the time steps $k_{n}=k$ for all $n$, and distinguish two cases. First, when $\tau / k$ is not an integer, then the $\mathrm{dG}(1)$ method is second order accurate, which is consistent with our result on the error estimate. When $\tau / k$ is, however, an integer, we observe a higher order accuracy of order three. Figure 3 shows both cases.

Second, an important result of the new dGcG-FEM algorithm is the successful treatment of integro-differential equations as

$$
\frac{\partial u}{\partial t}(t, x)+\alpha u(t, x)=\int_{\Omega} J(x, r) S(u(t, r)) d r, \quad \bar{\Omega}=[-1,1],
$$

i.e., when the delay is zero in the neural field equation. In our numerical simulation, we considered $J(x, r)=1$, and $S(u(t, r))=u(t, r), \alpha=1$, and $u_{0}(x)=x$. The exact 


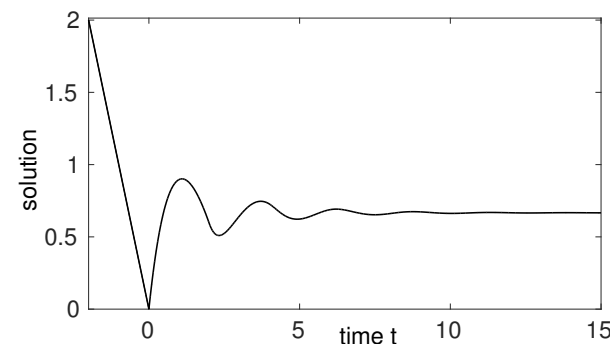

(a)

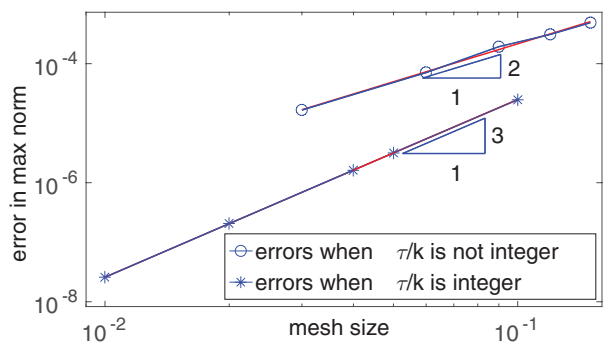

(b)

FIG. 3. (a) The $d G(1)$ solution of (52). (b) Discretization error in a log-log plot when $\tau / k$ is not an integer and when it is an integer.

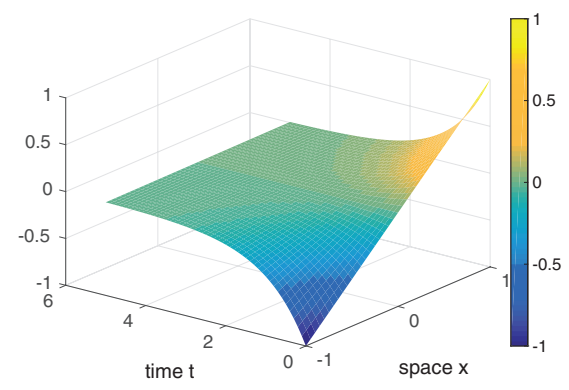

(a)

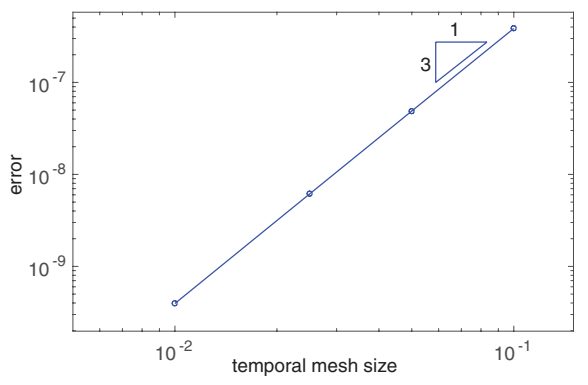

(b)

FIG. 4. (a) The $d G c G(1)$ solution of (53). (b) Discretization error in a log-log plot.

solution of (53) is $u(t, x)=x e^{-t}$, which converges to zero as $t \rightarrow \infty$ for every $x \in \Omega$. We compared the $\mathrm{dGcG(1)}$ method, using linear basis functions, both in space and time, with the exact solution and observed that the error is of superconvergent order; see Figure 4. In this example we also verified the order of accuracy in space.

5.2. Neural field equations. In this section we demonstrate the dGcG method in one and two space dimensions when using linear basis functions, both in space and time $(\mathrm{dGcG}(1))$. In our examples space and time are rescaled such that $\bar{\Omega}=[-1,1]^{d}$, $d=1,2$, and the propagation speed is 1 . The delay function is

$$
\tau(x, y)=\tau_{0}+\|x-y\|,
$$

where $\tau_{0} \geq 0$ is a fixed finite delay and in the two-dimensional case we will use both the $\|\cdot\|_{1}$-norm and the $\|\cdot\|_{2}$-norm. Note that the size of the maximal delay is different in the two norms considered. The activation function is

$$
S(u)=\frac{1}{1+e^{-\sigma u}}-\frac{1}{2} \forall u \in \mathbb{R} .
$$

5.2.1. Neural field equations in one space dimension. Consider first a single population model (2) when the space is one-dimensional,

$$
\frac{\partial u}{\partial t}(t, x)=-\alpha u(t, x)+\int_{-1}^{1} J(x, r) S(u(t-\tau(x, r), r)) d r
$$




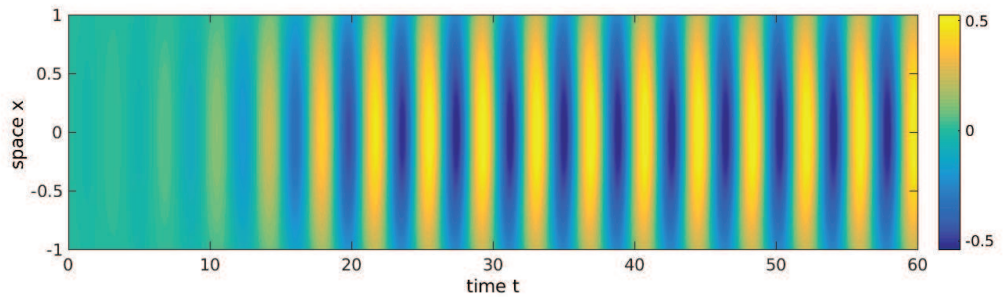

FIG. 5. Time evolution of system (56) in test case (1a) when $\sigma=6$, beyond a Hopf bifurcation.

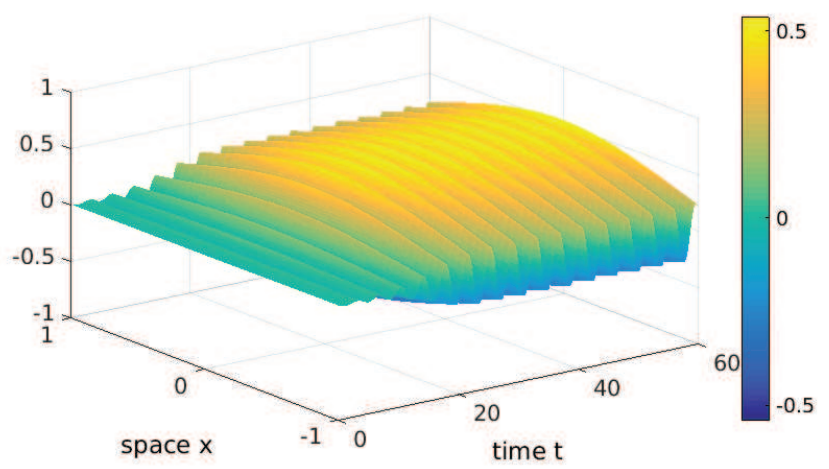

FIG. 6. Surface plot of the time evolution of the system (56) in test case (1a) when $\sigma=6$, beyond a Hopf bifurcation.

with

$$
\tau(x, y)=\tau_{0}+|x-y|
$$

Hopf bifurcations play an important role in the analysis of neural field equations. By choosing the steepness parameter $\sigma$ of the activation function as a bifurcation parameter, we can simulate, using the dGcG(1) scheme, the space-time evolution of the solution beyond a Hopf bifurcation. We choose the parameter $\alpha=1$ and the delay $\tau_{0}=1$. The initial function for these simulations is $u(s, x)=0.01, s \in\left[-\tau_{\max }, 0\right]$ and $x \in \bar{\Omega}$. Note that, because the size of the delay is relatively large compared to the time step, we do not need to linearize the system to solve the algebraic equations with a Newton method. To observe periodic oscillations, we distinguish two cases.

Test case (1a). Homogeneous kernel. As in [14], in this simulation the connectivity function has a bi-exponential form

$$
J(x, r)=\hat{J}(|x-r|)=\hat{c}_{1} e^{-\mu_{1}|x-r|}+\hat{c}_{2} e^{-\mu_{2}|x-r|}, \quad x, r \in[-1,1],
$$

with $\hat{c}_{1}=3.0, \hat{c}_{2}=-5.5, \mu_{1}=0.5, \mu_{2}=1.0$. The steepness of the activation function (55) is $\sigma=6$. Figure 5 shows the time evolution of the system, and Figure 6 is a surface plot of the numerical solution beyond a Hopf bifurcation.

Test case (1b). Neural fields with spatial inhomogeneity. Consider the locally changed connectivity

$$
\tilde{J}(x, y)=J(x, y)+\left.\omega J(x, y)\right|_{\tilde{\Omega}}, \quad \omega>0,
$$



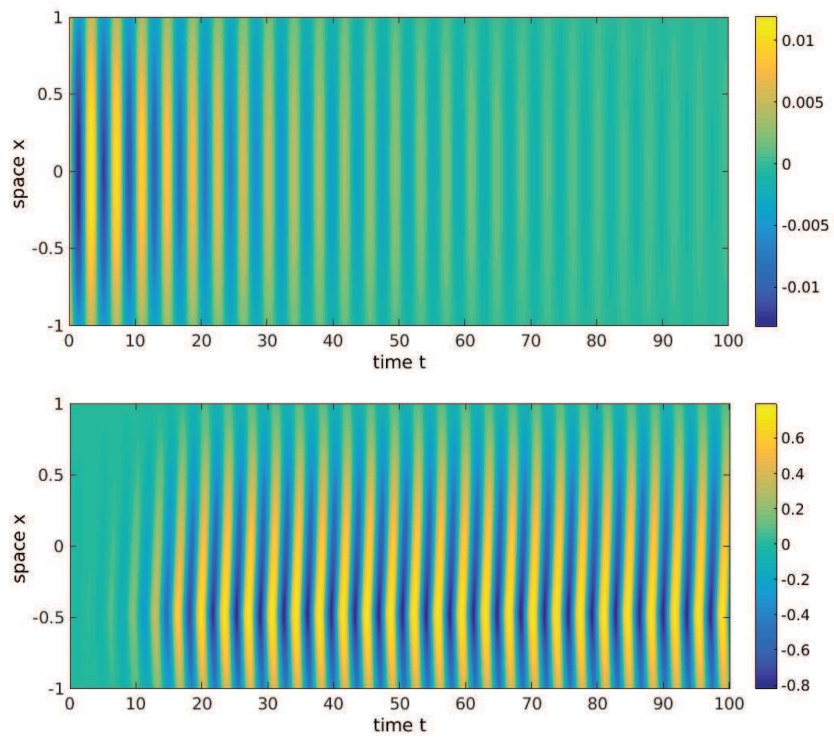

FIG. 7. Time evolution of system (56) in test case (1b) when $\sigma=4$, in the homogeneous (top) and the inhomogeneous (bottom) cases.

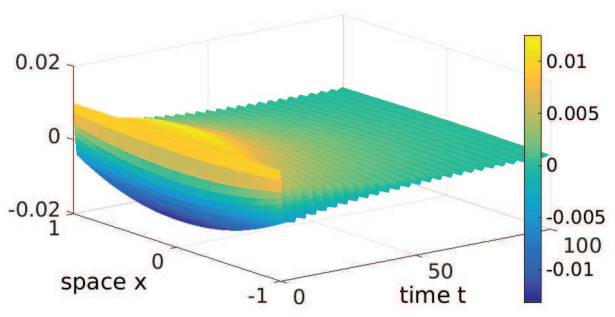

(a)

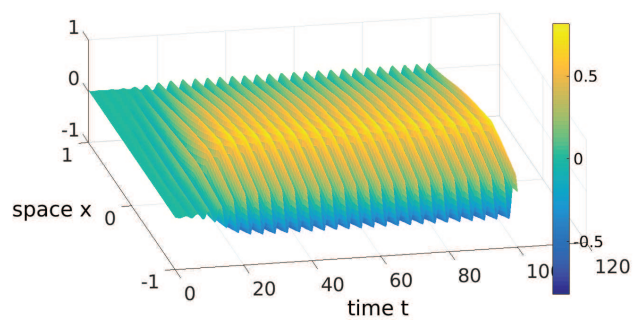

(b)

FIG. 8. Time evolution of system (56) in test case (1b) when $\sigma=4$, at given spatial position $x$, in the homogeneous (a) and the inhomogeneous (b) cases.

where $J$ is as given in (58) with the same parameters and $\tilde{\Omega} \subset \Omega$. This means that in $(56)$ the connectivity $J(x, y)$ is changed to $\tilde{J}(x, y)$. The activation function is given in (55) with the bifurcation parameter $\sigma=4$, chosen below the threshold for Hopf bifurcation to occur in the homogeneous case; see [14]. In Figures 7, 8, and 9 , we compare the solution of the system with homogeneous kernel, with the solution where we have locally changed the connectivity in one element $\tilde{\Omega}=K \in$ $\overline{\mathcal{T}}_{h}$. Our simulations show that while the solution converges to a steady state in the homogeneous case, in the inhomogeneous case the solution becomes periodic $(\omega=15)$. This is a new phenomenon observed in the one-dimensional case. It requires, however, further bifurcation analysis in the two-parameter space $(\sigma, \omega)$.

5.2.2. Neural field equations in two space dimensions. Although in the two-dimensional case there are few analytical results, our numerical computations show that with a proper choice of the parameters, we can observe similar phenomena as in the one-dimensional case. 


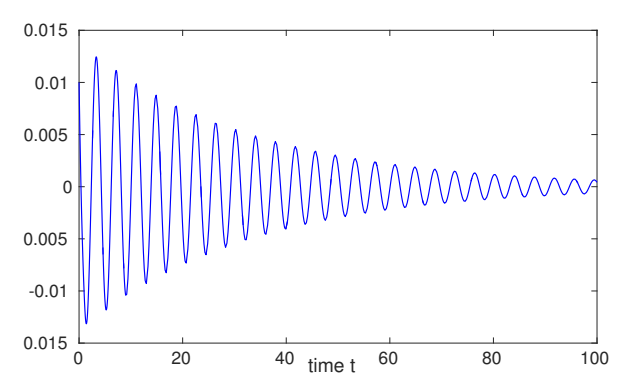

(a)

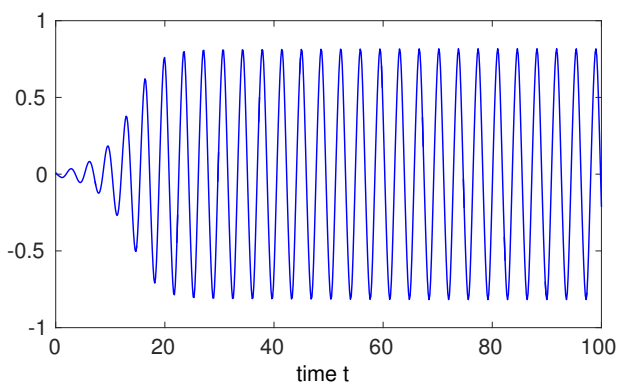

(b)

FIG. 9. Time evolution of system (56) in test case (1b) when $\sigma=4$, at given spatial position $x$, in the homogeneous (a) and the inhomogeneous (b) cases.

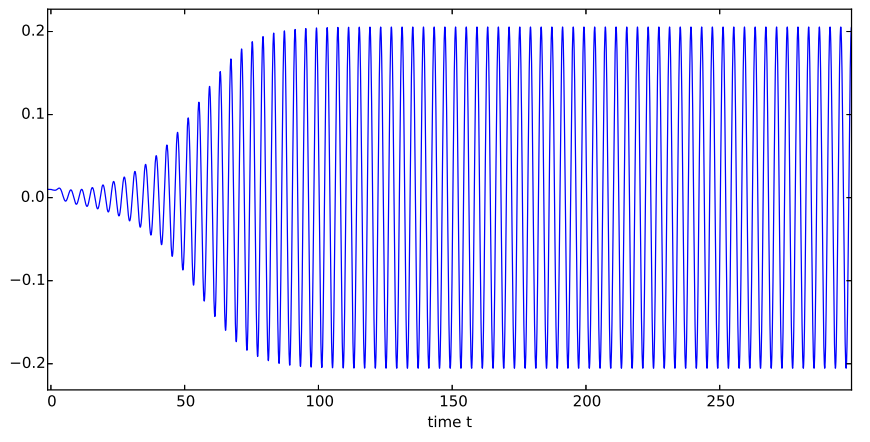

FIG. 10. Time evolution of the system in test case (2a) when $\sigma=6$, at a given position in space. (60)

Test case (2). First, we choose the connectivity function as

$J(x, r)=\hat{J}(\|x-r\|)=\hat{c}_{1} e^{-\mu_{1}\|x-r\|}+\hat{c}_{2} e^{-\mu_{2}\|x-r\|}, \quad \hat{c}_{j} \in \mathbb{R}, \mu_{j} \in \mathbb{R}, x, r \in[-1,1]^{2}$.

Test case (2a). Homogeneous kernel. As in the one-dimensional case, we fix the parameters $\hat{c}_{1}=3.0, \hat{c}_{2}=-5.5, \mu_{1}=0.5, \mu_{2}=1.0$. The rate of natural decay of activity $\alpha=1$, the delay $\tau_{0}=1$, and the initial function is again $u(s, x, y)=0.01$ for all $s \in\left[-\tau_{\max }, 0\right]$ and $x, y \in \bar{\Omega}$. When the steepness of the activation function is $\sigma=4$, the numerical solution converges to the zero steady state, while for $\sigma=6$ the solution becomes periodic in time for both norms. This periodic solution is plotted in Figures 10 and 11, where the $\|\cdot\|_{1}$-norm was used.

Test case (2b). Spatial inhomogeneity. We consider $\sigma=4$ and change locally the kernel (60) as in (59), where $\tilde{\Omega} \in \overline{\mathcal{T}}_{h}$ is a connected region in $\bar{\Omega}$ consisting of two space elements in our discretization and $\omega=30$. It is interesting to observe the same behavior as in the one-dimensional case, i.e., instead of convergence to zero, as in the homogeneous case, the solution becomes periodic; see Figure 12. The dynamic behavior of the solution is strongly influenced by the size of $\tilde{\Omega}$ and the magnitude of $\omega$. For a smaller region $\tilde{\Omega}$ or $\omega$ below our threshold, the oscillations are damped, and for larger values the amplitude of the oscillations grows. We used the $\|\cdot\|_{2}$-norm in this example.

Test case (3). In our second two-dimensional example, the connectivity kernel is 

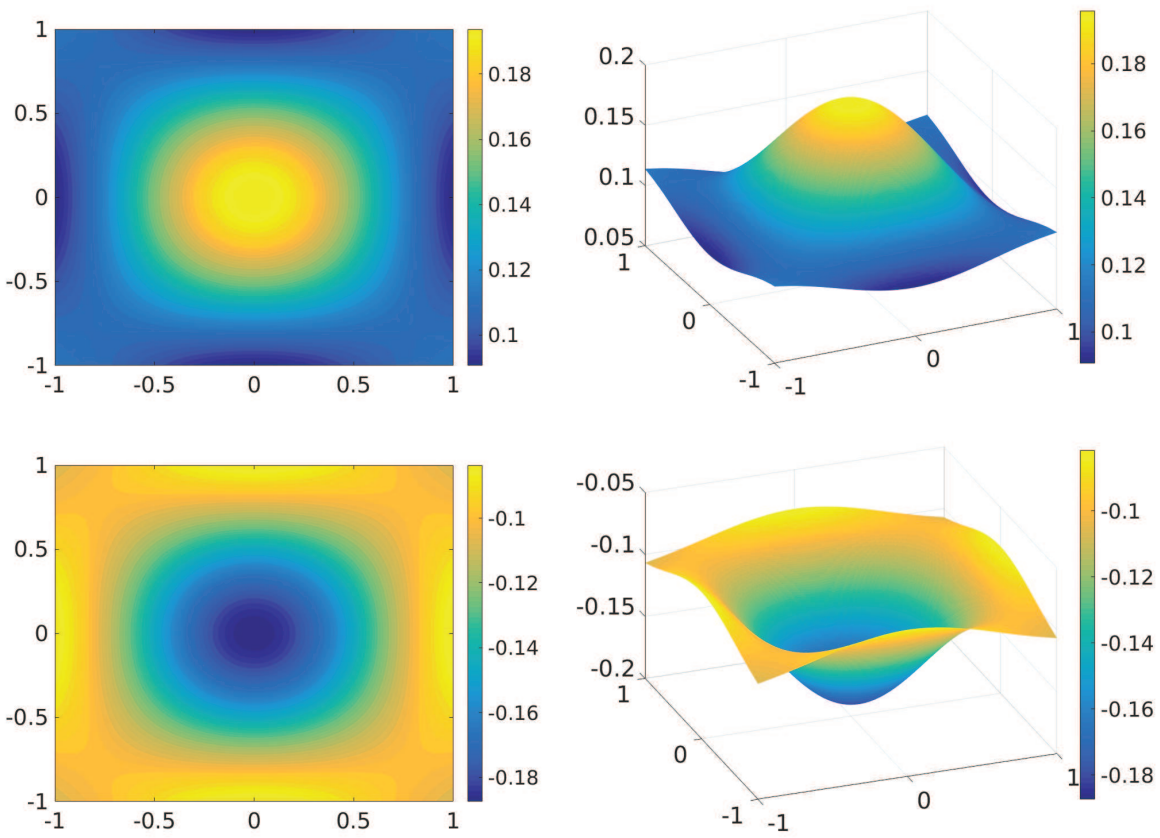

FIG. 11. The solution of the system in test case (2a) when $\sigma=6$, at the beginning and at half of a time period.
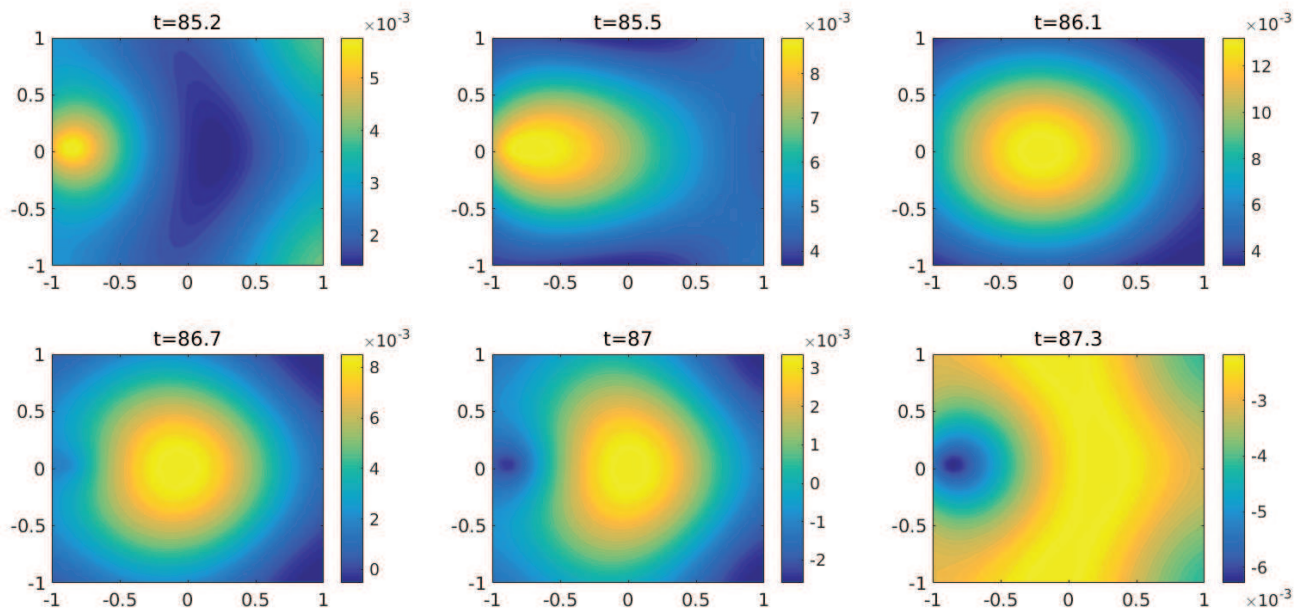

FIG. 12. Time evolution of the system in test case (2b) when $\sigma=4$, during half of a time period.

defined as in [8],

$$
J(x, r)=\hat{J}(\|x-r\|)=\frac{1}{\sqrt{2 \pi \xi_{1}^{2}}} e^{-\frac{\|x-r\|^{2}}{2 \xi_{1}^{2}}}-\frac{1}{\sqrt{2 \pi \xi_{2}^{2}}} e^{-\frac{\|x-r\|^{2}}{2 \xi_{2}^{2}}},
$$

with $\xi_{1}=0.3, \xi_{2}=0.4$, and we compute the numerical solutions when the norms are the $\|\cdot\|_{1}$ and $\|\cdot\|_{2}$ norms, respectively. The sigmoidal firing rate function is considered 

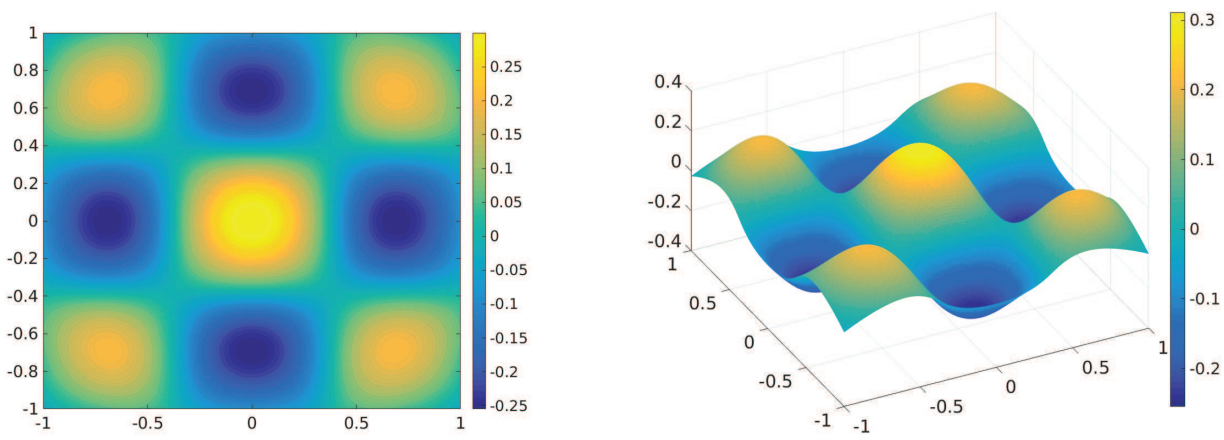

FIG. 13. Pattern emerging in the two-dimensional neural field model in test case (3a), with connectivity (61) and $\|\cdot\|_{1}$-norm.
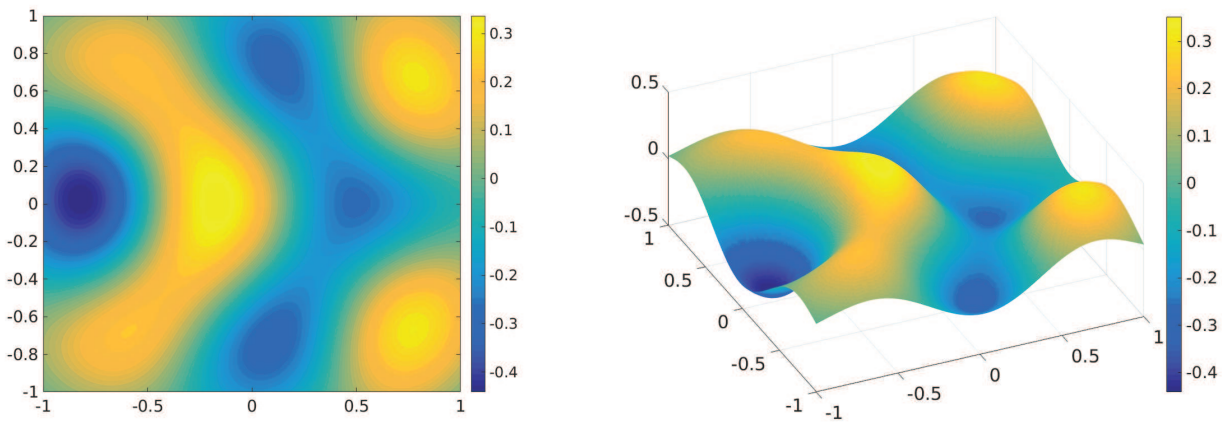

FIG. 14. Pattern emerging in the two-dimensional neural field model in test case (3b), with connectivity (61), added inhomogeneity, and $\|\cdot\|_{2}$-norm.

as in (55) with steepness $\sigma=45$. In this example, we choose the rate of natural decay of activity as a bifurcation parameter. When $\alpha=1$, the solution converges to the zero steady state, and when $\alpha=1 / 5$, we observe the formation of two different patterns for the homogeneous and inhomogeneous kernels, respectively.

Test case (3a). Homogeneous kernel. The initial function is chosen as $u_{0}(s, r)=$ $\cos (7 x) \cos (7 y), r=(x, y) \in \bar{\Omega}$ for all $s \in\left[-\tau_{\max }, 0\right]$. In Figure 13 we observe that the numerical solution is converging to a simple bi-periodic pattern. The norm in the connectivity and delay functions in this computation is the $\|\cdot\|_{1}$-norm, but a similar pattern can be observed in the $\|\cdot\|_{2}$-norm case.

Test case (3b). Spatial inhomogeneity. When we change the connectivity (61) locally to (59), with $\omega=15$, we observe the emergence of a different pattern than in the homogeneous case; see Figure 14. The initial function is the constant function $u_{0}(s, r)=0.01, r=(x, y) \in \bar{\Omega}$ for all $s \in\left[-\tau_{\max }, 0\right]$, and we used the $\|\cdot\|_{2}$-norm in this computation.

Finally, note that in all of our two-dimensional computations we used 1600 space elements.

6. Concluding remarks. In this paper we have presented a new space-time dGcG-FEM to solve delay integro-differential equations with space-dependent delays. The main result is an a priori error estimate of the space-time dGcG method, which 
also shows that the method is numerically stable. We demonstrated that by using a dGcG method we can handle general connectivity, synaptic activation, and delay functions, and we do not need to make any restriction on spatial dimension or shape of the domain. This makes it possible to extend our model to more general domains as well as more populations in the system, which are particularly interesting in applications.

Acknowledgment. We would like to thank Sid Visser for his great help with the two-dimensional computations.

\section{REFERENCES}

[1] A. Bellen and M. Zennaro, Numerical Methods for Delay Differential Equations, Oxford University Press, Oxford, UK, 2003.

[2] S. C. Brenner and L. R. Scott, The Mathematical Theory of Finite Element Methods, 2nd ed., Springer, New York, 1994.

[3] P. G. Ciarlet, The Finite Element Method for Elliptic Problems, North-Holland, Amsterdam, 1978.

[4] P. G. Ciarlet And P. A. RAViart, Interpolation theory over curved elements, with applications to finite element methods, Comput. Methods Appl. Mech. Engrg., 1 (1972), pp. 217-249.

[5] K. ERIKSSON AND C. Johnson, Error estimates and automatic time step control for nonlinear parabolic problems, I, SIAM J. Numer. Anal., 24 (1987), pp. 12-23, https://doi.org/10. $1137 / 0724002$.

[6] K. ERIKSSON AND C. Johnson, Adaptive finite element methods for parabolic problems V: Long-time integration, SIAM J. Numer. Anal., 32 (1995), pp. 1750-1763, https://doi.org/ $10.1137 / 0732079$.

[7] K. Eriksson, C. Johnson, and V. Thomée, Time discretization of parabolic problems by the discontinuous Galerkin method, RAIRO Anal. Numer., 19 (1985), pp. 611-643.

[8] G. Faye and O. Faugeras, Some theoretical and numerical results for delayed neural field equations, Phys. D, 239 (2010), pp. 561-578.

[9] T. J. R. Hughes And G. Hulbert, Space-time finite element methods for elastodynamics: Formulations and error estimates, Comput. Methods Appl. Mech. Engrg., 66 (1988), pp. 339363.

[10] A. Hutt And N. Rougier, Numerical simulation scheme of one-and two-dimensional neural fields involving space-dependent delays, in Neural Fields, Theory and Applications, Springer, New York, 2014, pp. 175-185.

[11] P. M. Lima ANd E. BuCKWAR, Numerical solution of the neural field equation in the twodimensional case, SIAM J. Sci. Comput., 37 (2015), pp. B962-B979, https://doi.org/10. $1137 / 15 \mathrm{M} 1022562$.

[12] V. ThoméE, Galerkin Finite Element Methods for Parabolic Problems, Springer, New York, 1997.

[13] J. J. W. van der Vegt and H. van der Ven, Space-time discontinuous Galerkin finite element method with dynamic grid motion for inviscid compressible flows. I. General formulation, J. Comput. Phys., 182 (2002), pp. 546-585.

[14] S. A. van Gils, S. G. Janssens, Y. A. Kuznetsov, and S. Visser, On local bifurcations in neural field models with transmission delays, J. Math. Biol., 66 (2013), pp. 837-887.

[15] R. Veltz and O. Faugeras, Stability of the stationary solutions of neural field equations with propagation delays, J. Math. Neurosci., 1 (2011), https://doi.org/10.1186/2190-8567-1-1.

Copyright $@$ by SIAM. Unauthorized reproduction of this article is prohibited. 